\title{
Rational blow-down along Wahl type plumbing trees of spheres
}

\author{
MARIA MiCHALOGIORGAKI
}

\begin{abstract}
In this article, we construct smooth 4-manifolds homeomorphic but not diffeomorphic to $\mathbb{C P}^{2} \# k \overline{\mathbb{C P}}^{2}$, for $k \in\{6,7,8,9\}$, using the technique of rational blow-down along Wahl type plumbing trees of spheres (see Wahl [17]).
\end{abstract}

57R55, 57R57; 14J26, 53D05

\section{Introduction}

Over the past three years, and due to examples constructed by Park [13], Fintushel and Stern [2], Stipsicz and Szabó [16], Park, Stipsicz and Szabó [14], and Ozsváth and Szabó [12], there has been renewed interest in the problem of finding the smallest $\mathrm{k}$ for which $\mathbb{C P}^{2} \# k \overline{\mathbb{C P}}^{2}$ admits an exotic smooth structure. All these examples are constructed using the rational blow-down operation along lens spaces.

In this paper, we study a more generalized rational blow-down operation along certain Seifert fibered 3-manifolds. This technique, for the case of Wahl type plumbing trees of spheres, together with knot surgery along a regular fiber in a double node neighborhood (see [2]), are then used to construct manifolds homeomorphic but not diffeomorphic to $\mathbb{C P}^{2} \# k \overline{\mathbb{C P}}^{2}$, for $k \in\{6,7,8,9\}$.

\section{Acknowledgements}

The author would like to thank Zoltán Szabó for his guidance and support while working on this problem. The author is indebted to him for sharing his ideas on the subject. The author would also like to thank András Stipsicz for useful suggestions on an earlier version of this article as well as the referee for a careful reading of the manuscript and several constructive comments. 


\section{Seiberg-Witten invariants and surgery along monopole $\mathbf{L}-$ spaces}

In this section, we provide a very brief review of the Seiberg-Witten theory of 4manifolds in general as well as in the special case $b_{2}^{+}=1$ and we study the effect of surgery along monopole L-spaces on Seiberg-Witten invariants, using monopole Floer homology. These will be the main tools for our constructions in the next sections. For more details, we refer the reader to Morgan [10], Scorpan [15], Fintushel and Stern [1], Kronheimer, Mrowka, Ozsváth and Szabó [9] and Kronheimer and Mrowka [8].

\subsection{Seiberg-Witten invariants}

Let $X$ be an oriented, closed, Riemannian 4-manifold and $\mathfrak{s}$ a $\operatorname{spin}^{c}$ structure on $X$. Suppose that $W_{\mathfrak{s}}^{+}$and $W_{\mathfrak{s}}^{-}$are the associated $U(2)$ spinor bundles and $L \rightarrow X$ with $L \simeq \operatorname{det} W_{\mathfrak{s}}^{+} \simeq \operatorname{det} W_{\mathfrak{s}}^{-}$is the associated determinant line bundle. Given a pair $(A, \Psi) \in A_{X}(L) \times \Gamma\left(W_{\mathfrak{s}}^{+}\right)$, where $A_{X}(L)$ denotes the space of connections on $L$, and a $g$-self-dual 2-form $\eta \in \Omega_{g}^{+}(X, \mathbb{R})$, the perturbed Seiberg-Witten equations are

$$
D_{A} \Psi=0, \quad F_{A}^{+}=i\left(\Psi \otimes \Psi^{*}\right)_{o}+i \eta
$$

where $D_{A}: \Gamma\left(W_{\mathfrak{s}}^{+}\right) \rightarrow \Gamma\left(W_{\mathfrak{s}}^{-}\right)$is the Dirac operator and $\left(\Psi \otimes \Psi^{*}\right)_{o}$ is the trace free part of the ednomorphism $\Psi \otimes \Psi^{*}$.

The quotient of the solution space to the equations above under the action of the gauge $\operatorname{group} \operatorname{Aut}(L)=\operatorname{Map}\left(X, S^{1}\right)$, denoted here by $M_{X}(L)$, has formal dimension

$$
\operatorname{dim} M_{X}(L)=\frac{1}{4}\left(c_{1}(L)^{2}-(3 \operatorname{sign}(X)+2 e(X))\right)
$$

Under the additional assumption that $b_{2}^{+}>0$ and for generic form $\eta, M_{X}(L)$ is a smooth compact manifold, since when $b_{2}^{+}>0$ there are no reducible solutions, that is, no singularities in the quotient space.

The Seiberg-Witten invariant for $X$ is a function $S W_{X}: \operatorname{Spin}^{c}(X) \rightarrow \mathbb{Z}$ defined as follows:

- If $\operatorname{dim} M_{X}(L)<0$ or odd, then $S W_{X}(L)=0$.

- If $\operatorname{dim} M_{X}(L)=0$, then $S W_{X}(L)=$ number of points in $M_{X}(L)$, counted with sign.

- If $\operatorname{dim} M_{X}(L)=2 n>0$, then $S W_{X}(L)=\left\langle\mu^{n},\left[M_{X}(L)\right]\right\rangle$, where the element $\mu \in H^{2}\left(M_{X}(L) ; \mathbb{Z}\right)$ is the Euler class of the basepoint map

$$
\widetilde{M}_{X}(L)=\{\operatorname{solutions}(A, \Psi)\} / \operatorname{Aut}^{o}(L) \rightarrow M_{X}(L),
$$

Algebraic $8 \mathcal{G}$ Geometric Topology, Volume 7 (2007) 
which is an $S^{1}$ fibration if there are no reducible solutions. Here,

$\operatorname{Aut}^{o}(L)=\{$ gauge transformations which are the identity

on the fiber of $L$ over a fixed basepoint on $X$ \}.

$S W_{X}$ is independent of $g$ and $\eta$ provided that $b_{2}^{+}>1$. In the case $b_{2}^{+}(X)=1$, there is a codimension-one submanifold of metrics for which there are reducible solutions and this must be excluded. Then the SW invariant of a given $\operatorname{spin}_{c}$ structure has two values, depending on the metric, and the wall-crossing formula describes the relation between these values.

Wall-crossing formula Suppose that $X$ is a closed, oriented 4-manifold which has $b_{2}^{+}(X)=1, H_{1}(X ; \mathbb{Z})=0$ and a fixed orientation for $H_{+}^{2}(X ; \mathbb{R}), \mathfrak{s}$ is a $\operatorname{spin}^{c}$ structure on $X$ such that $c_{1}(L) \neq 0, R$ is the space of Riemannian metrics $g$ on $X, \omega^{+}(g)$ is the $g$-self-dual harmonic form of norm one which lies in the positive component of $H_{2}^{+}(X ; \mathbb{R})$ as measured by the given orientation and $R^{+}=\left\{g \in R / \omega^{+}(g) \cdot c_{1}(L)>0\right\}$, $R^{-}=\left\{g \in R / \omega^{+}(g) \cdot c_{1}(L)<0\right\}$. Then for all $g \in R^{+} \sqcup R^{-}, S W_{g}(\mathfrak{s})$ is defined and assuming that $d(\mathfrak{s})=\operatorname{dim} M_{X}(L) \geq 0$ and even

$$
S W_{+}(\mathfrak{s})=S W_{-}(\mathfrak{s})-(-1)^{d(\mathfrak{s}) / 2} .
$$

Here, $S W_{+(-)}(\mathfrak{s})$ denotes the constant value of $S W_{g}(\mathfrak{s})$ on $R_{+(-)}$respectively.

\subsection{Monopole Floer homology}

We now provide a very brief review of monopole Floer homology as constructed by P Kronheimer and T Mrowka. We refer the reader to Kronheimer, Mrowka, Ozsváth and Szabó [9] and to Kronheimer and Mrowka [8] for more details and point out that this version of Floer homology is conjectured to be isomorphic to Heegaard Floer homology.

Let $Y$ be a smooth, oriented, compact, connected 3-manifold without boundary. To it, there are associated three vector spaces over a field $\mathbb{F}$, namely $\widetilde{H M}_{\bullet}(Y), \widehat{H M} \bullet(Y)$ and $\overline{H M}_{\bullet}(Y)$. These spaces, called Floer homology groups, come equipped with linear maps $i_{*}, j_{*}$ and $p_{*}$ which form a long exact sequence

$$
\cdots \stackrel{i_{*}}{\rightarrow} \widetilde{H M} \bullet(Y) \stackrel{j_{*}}{\rightarrow} \widehat{H M} \bullet(Y) \stackrel{p_{*}}{\longrightarrow} \overline{H M} \bullet(Y) \stackrel{i_{*}}{\rightarrow} \widetilde{H M} \bullet(Y) \stackrel{j_{*}}{\rightarrow} \cdots
$$

and with an endomorphism $u$ of degree -2 that makes the three spaces modules over the polynomial ring $\mathbb{F}[u]$. 
In addition, to each cobordism $W: Y_{0} \rightarrow Y_{1}$, there are associated maps

$$
\begin{aligned}
& \widetilde{H M}(W): \widetilde{H M} \bullet\left(Y_{0}\right) \rightarrow \widetilde{H M} \bullet\left(Y_{1}\right), \\
& \widehat{H M}(W): \widehat{H M}_{\bullet}\left(Y_{0}\right) \rightarrow \widehat{H M}_{\bullet}\left(Y_{1}\right), \\
& \overline{H M}(W): \overline{H M}_{\bullet}\left(Y_{0}\right) \rightarrow \overline{H M}_{\bullet}\left(Y_{1}\right)
\end{aligned}
$$

for which $i_{*}, j_{*}, p_{*}$ give natural transformations. These maps respect the module structure of the Floer groups.

In this setting, we have the following

Definition 1 A rational homology 3-sphere Y for which $j_{*}: \widetilde{H M} \bullet(Y) \rightarrow \widehat{H M} \bullet(Y)$ is trivial is called a monopole $L-$ space.

\subsection{Using monopole Floer homology to compute Seiberg-Witten invari- ants after surgery along monopole $\mathrm{L}$-spaces}

After recalling some basics of Seiberg-Witten theory and Monopole Floer homology, we move on to compute how SW invariants change under surgery along monopole L-spaces.

Suppose $X$ is a 4-manifold decomposed into two pieces $Z$ and $P$ along a monopole L-space $Y$, with $P$ negative definite, and $\mathfrak{s} \in \operatorname{Spin}^{c}(X)$ (See Figure 1). Consider $B$ another negative definite 4 -manifold bounded by $Y$ such that $\mathfrak{s} \mid Z$ extends to $B$ and replace $P$ with $B$ in $X$ to get $X^{\prime}=Z \bigcup_{Y} B$ (See Figure 2). Denote the $\operatorname{spin}^{c}$ structure on $X^{\prime}$ by $\mathfrak{s}^{\prime}$. We would like to compute the change in SW invariants after such an operation. To this end, we study these configurations, using properties of monopole Floer homology. Most of the statements we make here are discussed in [8] and [9].

Remark 2 Note that our constructions in this paper (see next section) are special cases of the above, for $B$ rational balls and $P$ Wahl-type plumbing trees of spheres. The boundaries of the latter were proven to be monopole L-spaces in [9].

Our goal in this subsection is to prove that

Theorem 3 Suppose $Y$ is a monopole $L$-space, $H_{1}(Y)$ is finite, $P$ and $B$ are negative definite 4-manifolds with $b_{1}=0$ and $X=Z \bigcup_{Y} P, X^{\prime}=Z \bigcup_{Y} B$, for some 4-manifold $Z$. If $\mathfrak{s} \in \operatorname{Spin}^{c}(X), \mathfrak{s}^{\prime} \in \operatorname{Spin}^{c}\left(X^{\prime}\right), d(\mathfrak{s}), d\left(\mathfrak{s}^{\prime}\right) \geq 0$ and $\left.\mathfrak{s}\right|_{Z}=\left.\mathfrak{s}^{\prime}\right|_{Z}$, then $S W_{X}(\mathfrak{s})=S W_{X^{\prime}}\left(\mathfrak{s}^{\prime}\right)$.

In the case $b_{2}^{+}(X)=1, S W_{X, a_{1}}(\mathfrak{s})=S W_{X^{\prime}, a_{2}}\left(\mathfrak{s}^{\prime}\right)$, where $a_{1} \in H_{2}(X ; \mathbb{Z}), a_{2} \in$ $H_{2}\left(X^{\prime} ; \mathbb{Z}\right),\left.a_{1}\right|_{P}=\left.a_{2}\right|_{B}=0$ and $\left.a_{1}\right|_{Z}=\left.a_{2}\right|_{Z}$. 


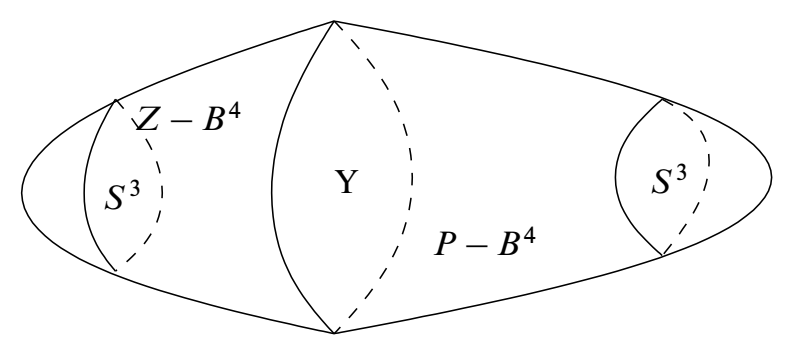

Figure 1: $X=Z \bigcup_{Y} P$

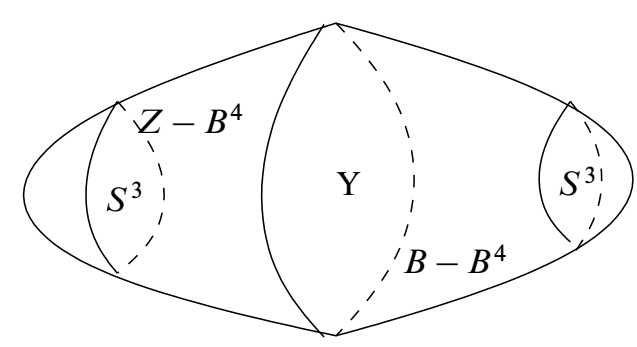

Figure 2: $X^{\prime}=Z \bigcup_{Y} B$

Proof Denote by $W, W_{1}$ and $W_{2}$ the cobordisms $Z-B^{4}: S^{3} \rightarrow Y, P-B^{4}: Y \rightarrow S^{3}$, $B-B^{4}: Y \rightarrow S^{3}$ respectively and $\mathfrak{s}_{1}=\left.\mathfrak{s}\right|_{W_{1}}, \mathfrak{s}_{2}=\left.\mathfrak{s}^{\prime}\right|_{W_{2}}$.

According to [9, Proposition 2.6], the fact that $Y$ is a rational homology sphere implies that

$$
\overline{H M} \bullet\left(Y,\left.\mathfrak{s}\right|_{Y}\right) \simeq \mathbb{F}\left[u^{-1}, u \rrbracket \text { as topological } F \llbracket u \rrbracket\right. \text { modules. }
$$

Here $\mathbb{E}\left[u^{-1}, u \rrbracket\right.$ denotes Laurent series finite in the negative direction. In addition, the long exact sequence (4) gives the exact sequence

$$
0 \rightarrow \widehat{H M} \bullet(Y) \stackrel{p_{*}}{\rightarrow} \overline{H M} \bullet(Y) \stackrel{i_{*}}{\rightarrow} \widetilde{H M} \bullet(Y) \rightarrow 0
$$

for $Y$ monopole L-space (see Definition 1). Combining (5) and (6), we get that the sequences

$$
0 \rightarrow \widehat{H M} \bullet\left(Y,\left.\mathfrak{s}\right|_{Y}\right) \stackrel{p_{*}}{\rightarrow} \overline{H M} \bullet\left(Y,\left.\mathfrak{s}\right|_{Y}\right) \stackrel{i_{*}}{\rightarrow} \widetilde{H M} \bullet\left(Y,\left.\mathfrak{s}\right|_{Y}\right) \rightarrow 0
$$

and

$$
0 \rightarrow \mathbb{F} \llbracket u \rrbracket \rightarrow \mathbb{F}\left[u^{-1}, u \rrbracket \rightarrow \mathbb{F}\left[u^{-1}, u \rrbracket / \mathbb{E} \llbracket u \rrbracket \rightarrow 0\right.\right.
$$

are isomorphic as sequences of topological $\mathbb{E} \llbracket u \rrbracket$ modules. The corresponding isomorphism of short exact sequences holds if we consider $S^{3}$ instead of $Y$, because $S^{3}$ is a 
monopole L-space as a 3-manifold with positive scalar curvature. Such 3-manifolds were proven to be monopole L-spaces in [9].

We will now use $\overrightarrow{H M}$. as defined in [8]: For $Y_{0}, Y_{1}$ compact, connected, oriented 3-manifolds and $W$ isomorphism class of connected cobordisms equipped with an homology orientation, $\overrightarrow{H M}_{\bullet}(W): \widehat{H M}_{\bullet}\left(Y_{0}\right) \rightarrow \widehat{H M}_{\bullet}\left(Y_{1}\right)$ is a (canonical choice of) map such that the diagram

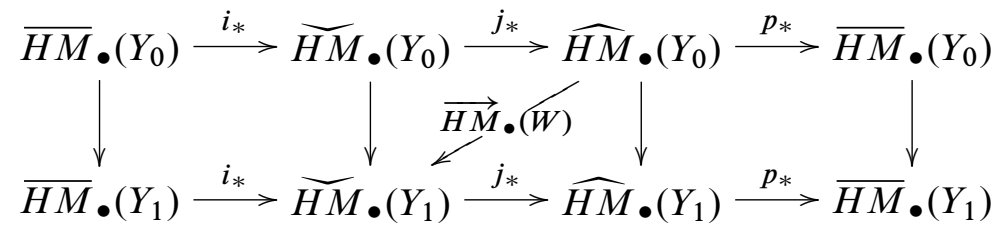

commutes. For the special case of a cobordism where $W$ is the complement of two disjoint balls in a closed, oriented manifold $X$ viewed as a cobordism $W: S^{3} \rightarrow S^{3}$, [8, Proposition 3.6.1] states that the sum of the SW invariants of the 4-manifold $X$ is determined by the map $\overrightarrow{H M} \bullet(W)$. Even more, using local coefficients, it can be proven that the individual SW invariants are determined by the same map [8, Proposition 3.8.1]. Applying the composition laws that $\overrightarrow{H M} \bullet$ satisfies according to [8] to our cobordisms $W, W_{1}$ and $W_{2}$ gives that $\overrightarrow{H M} \bullet\left(W_{i} \circ W\right)=\widetilde{H M} \bullet\left(W_{i}\right) \circ \overrightarrow{H M} \bullet(W), i \in\{1,2\}$. In a more refined version, for our fixed $\operatorname{spin}^{c}$ structures $\mathfrak{s}$ and $\mathfrak{s}_{\mathfrak{i}}$ on $W$ and $W_{i}$ respectively, we have that

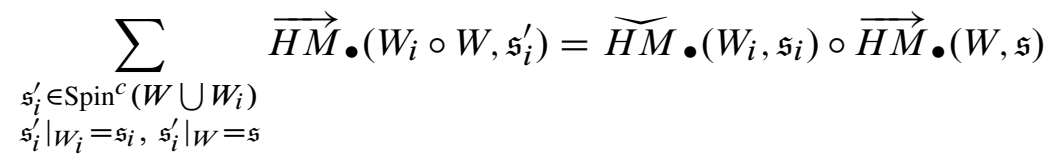

This sum contains precisely one term, since $Y$ is a rational homology 3-sphere and so, (7) gives that

$$
\overrightarrow{H M}_{\bullet}\left(W_{1} \circ W, \mathfrak{s}\right)=\widetilde{H M} \bullet\left(W_{1}, \mathfrak{s}_{1}\right) \circ \overrightarrow{H M} \bullet(W, \mathfrak{s})
$$

and

$$
\overrightarrow{H M} \bullet\left(W_{2} \circ W, \mathfrak{s}^{\prime}\right)=\widetilde{H M} \bullet\left(W_{2}, \mathfrak{s}_{2}\right) \circ \overrightarrow{H M} \bullet(W, \mathfrak{s})
$$

Taking into account that $\overrightarrow{H M} \bullet\left(W_{1} \circ W, \mathfrak{s}\right), \overrightarrow{H M} \bullet\left(W_{2} \circ W, \mathfrak{s}^{\prime}\right)$ determine $S W_{X}(\mathfrak{s})$ and $S W_{X^{\prime}}\left(\mathfrak{s}^{\prime}\right)$ respectively, it suffices to show that the maps $\widetilde{H M} \bullet\left(W_{1}, \mathfrak{s}_{1}\right)$ and $\overrightarrow{H M} \bullet\left(W_{2}, \mathfrak{s}_{2}\right)$ are isomorphisms in the range of $\overrightarrow{H M} \bullet(W, \mathfrak{s})$ to finish the proof. 
To see this, consider the diagram

$$
\begin{aligned}
\cdots \longrightarrow & \overline{H M} \bullet\left(Y,\left.\mathfrak{s}\right|_{Y}\right) \stackrel{i_{*}}{\longrightarrow} \widetilde{H M} \bullet\left(Y,\left.\mathfrak{s}\right|_{Y}\right) \longrightarrow 0 \\
\overline{H M} \bullet\left(W_{i}, \mathfrak{s}_{i}\right) \downarrow & \widetilde{H M} \bullet\left(W_{i}, \mathfrak{s}_{i}\right) \downarrow \\
\cdots \longrightarrow & \longrightarrow \overline{H M}_{\bullet}\left(S^{3},\left.\mathfrak{s}_{i}\right|_{S^{3}}\right) \stackrel{i_{*}}{\longrightarrow} \widetilde{H M} \bullet\left(S^{3},\left.\mathfrak{s}_{i}\right|_{S^{3}}\right) \longrightarrow
\end{aligned}
$$

where $i \in\{1,2\}$. We have assumed that $b_{1}\left(W_{i}\right)=0$ and $W_{i}$ is negative definite and under these assumptions the map $\overline{H M}\left(W_{i}, \mathfrak{s}_{i}\right): \overline{H M}_{j_{0_{i}}}(Y) \rightarrow \overline{H M}_{j_{1_{i}}}\left(S^{3}\right), i \in\{1,2\}$, where $j_{0_{i}} \in J\left(Y, \mathfrak{s}_{i} \mid Y\right)=$ \{homotopy classes of oriented 2-plane fields on $Y$ that determine the $\operatorname{spin}^{c}$ structure $\mathfrak{s}_{i} \mid Y$ on $\left.Y\right\}, j_{1_{i}} \in J\left(S^{3}, \mathfrak{s}_{i} \mid S^{3}\right)$ and $j_{0_{i}} \stackrel{\mathfrak{s}_{i}}{\sim} j_{1_{i}}$, is an isomorphism, as was proven in [9]. This implies that $\overline{H M} \bullet\left(W_{i}, \mathfrak{s}_{i}\right)$ is an isomorphism on the range of $\overrightarrow{H M} \bullet(W, \mathfrak{s})$ in the case where $d(\mathfrak{s}), d\left(\mathfrak{s}^{\prime}\right) \geq 0$.

In the case $b_{2}^{+}(X)=1$, the SW invariants depend on the choice of metric $g$ and perturbation $\eta$. If $b_{2}^{+}(W)=1$, then a choice of $g$ and $\eta$ for $W$ determines the chamber that will be used for our computations.

\section{The topological constructions}

To construct our 4-manifolds, we will blow-down certain Wahl type plumbing trees of spheres in rational surfaces. In order to locate these configurations in such surfaces, we use specific elliptic fibrations of $E(1)$ in each case. Proofs for the existence of such fibrations are postponed until the last section of our article.

\subsection{An exotic smooth structure on $\mathbb{C P}^{2} \# 9 \overline{\mathbb{C} \mathbb{P}^{2}}$}

Our first construction relies on the following existence result.

Proposition 4 There is an elliptic fibration of $E(1) \rightarrow \mathbb{C P}^{1}$ with a singular $I_{3}$ fiber, 9 fishtail fibers and one section.

Proof An outline of the proof of this proposition is provided in the appendix.

Consider the plumbing tree of spheres

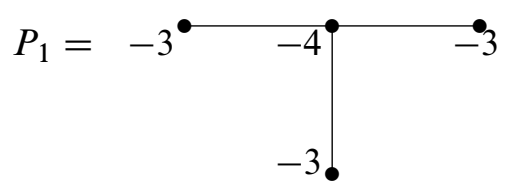


where dots represent disk bundles over $S^{2}$, numbers assigned to them refer to the corresponding Euler numbers and edges stand for plumbing connections and call $Y_{1}$ the boundary of $P_{1}$, that is, $Y_{1}=\partial P_{1}$. Proposition 5 below provides an embedding of $P_{1}$ into $\mathbb{C P}^{2} \# 13 \overline{\mathbb{C P}}^{2}$.

Proposition $5 \quad P_{1}$ embeds into $\mathbb{C P}^{2} \# 13 \overline{\mathbb{C P}}^{2}$.

Proof We use the fibration of Proposition 4 and in particular the $I_{3}$ fiber and one fishtail fiber, call it $F_{1}$, together with the section $E_{3}$. We blow up the double point of $F_{1}$ and denote the exceptional sphere by $E_{10}$. We further blow up at three points of the $I_{3}$ fiber, two of them on the -2 sphere intersecting the section $\left(E_{12}\right.$ and $\left.E_{13}\right)$ and the third at the intersection of the remaining -2 spheres $\left(E_{11}\right)$. Finally, we smooth the transverse intersection of $F_{1}$ with $E_{3}$. The above procedure, the outcome of which is depicted in Figure 3, provides the desired embedding.

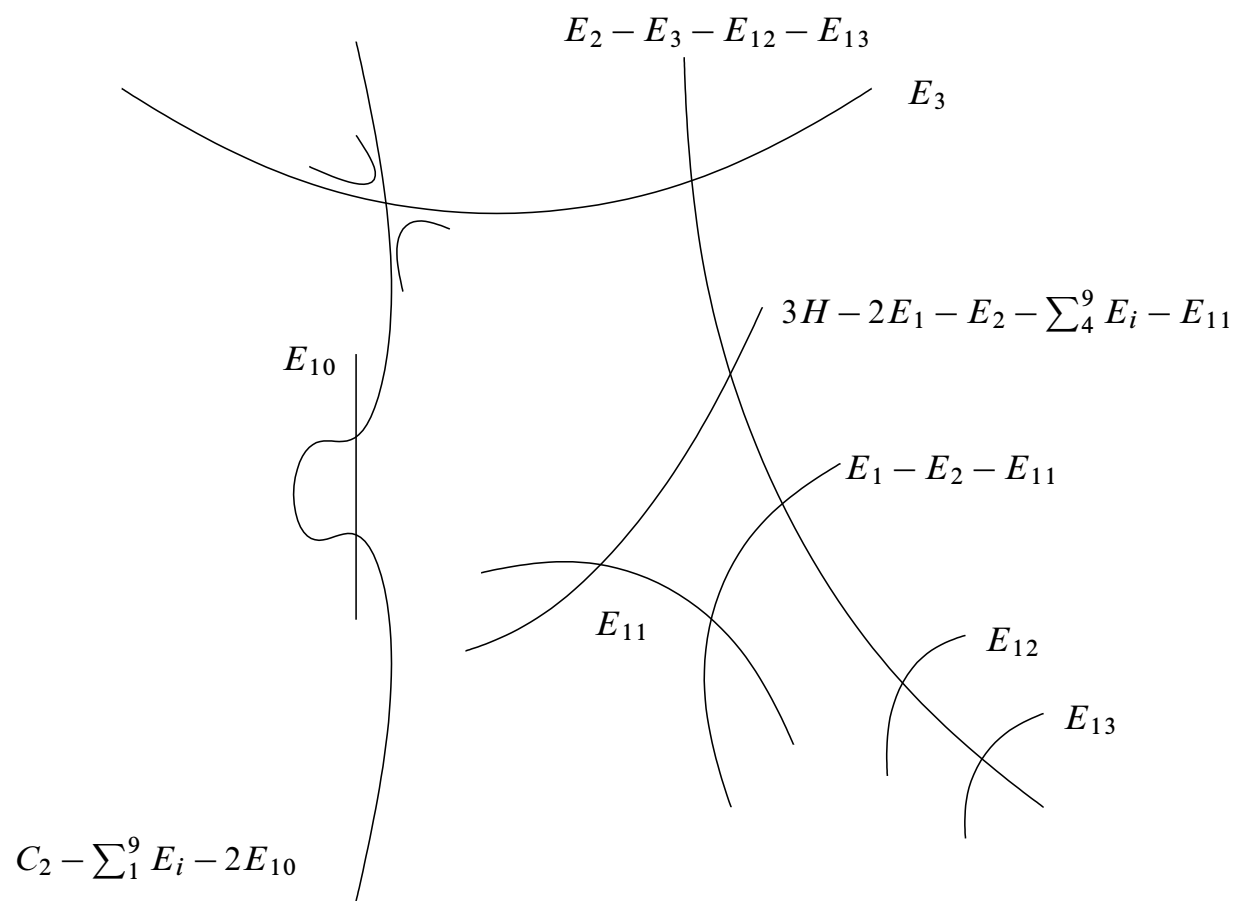

Figure 3: $P_{1}$ in $\mathbb{C P}^{2} \# 13 \overline{\mathbb{C P}}^{2}$

Remark 6 It is not hard to see that $Y_{1}$ bounds a rational homology ball $B_{1}$. To this end, we can use the fact that $P_{1}$ embeds in $\# 4 \overline{\mathbb{C P}}^{2}$ (See Figure 4). The closure of the 
complement of this embedding with reversed orientation is a rational ball. Alternatively, we can use Neumann [11] and construct such a rational ball explicitly.

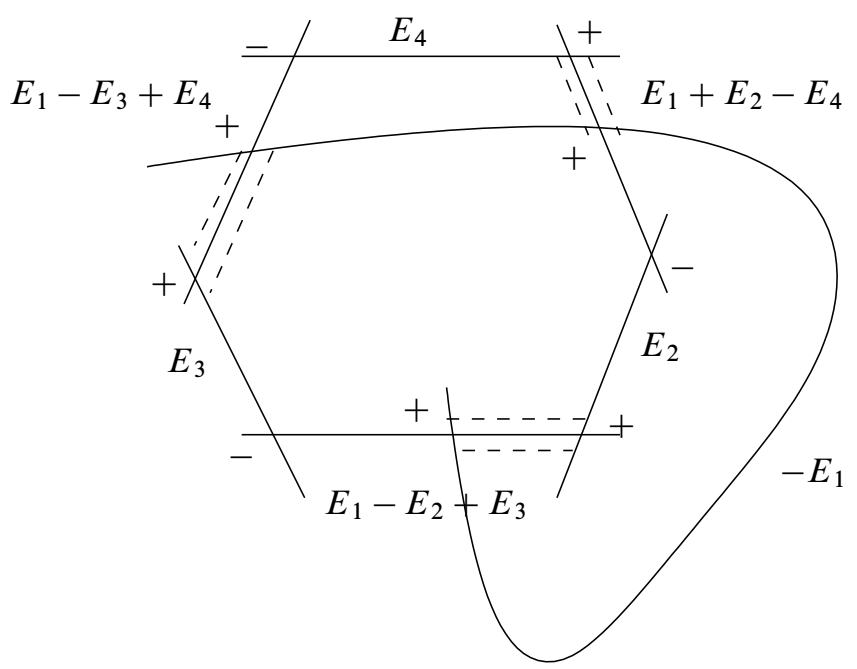

Figure 4: $\quad P_{1}$ in $\# 4 \overline{\mathbb{C P}}^{2}$

Theorem $7 \quad X_{1}^{\prime}=\left(\mathbb{C P}^{2} \# 13 \overline{\mathbb{C P}}{ }^{2}-\operatorname{int}\left(P_{1}\right)\right) \bigcup_{Y_{1}} B_{1}$ is homeomorphic to $\mathbb{C P}^{2} \# 9 \overline{\mathbb{C P}}{ }^{2}$.

Proof First, we will prove that $X_{1}^{\prime}$ is simply connected. The embedding of $P_{1}$ into $\mathbb{C P}^{2} \# 13 \overline{\mathbb{C P}}^{2}$ constructed above has a simply connected complement since the circles in the boundary $Y_{1}$ of the complement are homotopically trivial in the complement(here we are using the fact that rational surfaces are simply connected). In fact, it suffices to prove this for the normal circles to the -3 framed spheres $C_{2}-E_{1}-E_{2}-\sum_{4}^{9} E_{i}-2 E_{10}$ and $3 H-2 E_{1}-E_{2}-\sum_{4}^{9} E_{i}-E_{11}$ and this can be done easily using disks in one fishtail fiber and $E_{7}$ respectively. In addition, the map $\pi_{1}\left(\partial B_{1}\right) \rightarrow \pi_{1}\left(B_{1}\right)$ induced by the natural embedding is surjective, as one can see applying Van Kampen's Theorem for the decomposition $\# 4 \overline{\mathbb{C P}}{ }^{2}=P_{1} \bigcup_{Y_{1}}\left(\# 4 \overline{\mathbb{C P}}^{2} \backslash P_{1}\right)=P_{1} \bigcup_{\partial B_{1}} \bar{B}_{1}$, where $\bar{B}_{1}$ denotes $B_{1}$ with opposite orientation. Thus, $X_{1}^{\prime}$ is indeed simply connected. Now the statement of the theorem follows from Freedman's Theorem [3], after computing the Euler characteristic and the signature of the two manifolds.

\subsection{An exotic smooth structure on $\mathbb{C P}^{2} \# \mathbf{\overline { \mathbb { C P } }} \overline{\mathbb{P}}^{2}$.}

For our second construction, we will make use of the existence result stated in Proposition 8: 
Proposition 8 There is an elliptic fibration of $E(1) \rightarrow \mathbb{C P}^{1}$ with a singular $I_{5}$ fiber, 7 fishtail fibers and one section.

See Appendix A for a brief discussion of this.

Let $P_{2}$ denote the plumbing tree of spheres

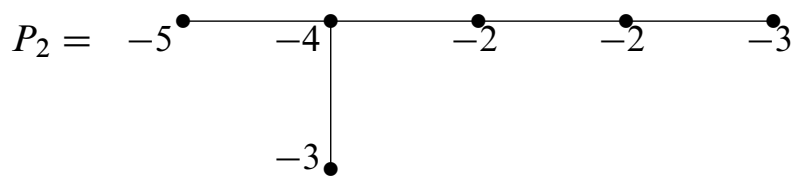

and call $Y_{2}$ the boundary of $P_{2}$. It is not hard to see that

Proposition $9 P_{2}$ embeds into $\mathbb{C P} \mathbb{P}^{2} \# 14 \overline{\mathbb{C P}}^{2}$.

Proof Consider the fibration of Proposition 8. Blow up two double points in two of the fishtail fibers. Then perform three further blow-ups at the $I_{5}$ fiber, one at the intersection point of two -2 spheres and the other two on the -2 sphere intersecting the section. Finally, smooth out the intersections of the two fishtails with the section to get the desired embedding.

Remark 10 To prove that $P_{2}$ bounds a rational homology ball, we will once again use an appropriate embedding of this manifold, that is, the embedding of $P_{2}$ in $\# 6 \overline{\mathbb{C P}}^{2}$.

Theorem $11 X_{2}^{\prime}=\left(\mathbb{C P}^{2} \# 14 \overline{\mathbb{C P}}^{2}-\operatorname{int}\left(P_{2}\right)\right) \bigcup_{Y_{2}} B_{2}$ is homeomorphic to $\mathbb{C P}^{2} \# 8 \overline{\mathbb{C P}}^{2}$.

Proof Simple connectivity of the complement of $P_{2}$ in $\mathbb{C P}^{2} \# 14 \overline{\mathbb{C P}}^{2}$ can be proven using disks in a fishtail fiber and some of the exceptional spheres. In addition to that, surjectivity of the map $\pi_{1}\left(\partial B_{2}\right) \rightarrow \pi_{1}\left(B_{2}\right)$ induced by the natural embedding follows from an application of Van Kampen's theorem for $\# 6 \overline{\mathbb{C P}}^{2}$, completely analogous to the one in Claim 2 in the proof of Theorem 7. These facts, together with Freedman's classification theorem, lead to the proof of the theorem.

We would here like to point out that the plumbing trees of spheres we have used so far, that is, $P_{1}$ and $P_{2}$, belong in the category of manifolds studied by Neumann in [11]. In his notation $P_{1} \sim M(0 ;(1,1),(3,2),(3,2),(3,2))(p=q=r=2)$ and $P_{2} \sim M(0 ;(1,1),(3,2),(5,4),(5,2))(p=q=2, r=4)$. In addition, note that using Neumann's results in this paper, one can find rational balls bounded by $\partial P_{i}, i=1,2$, with known handlebody decompositions.

For our next two constructions, we will need to combine the techniques used above with knot surgery in a double node neighborhood, as it was introduced in [2] by $\mathrm{R}$ Fintushel and R Stern. 


\subsection{An exotic smooth structure on $\mathbb{C P}^{2} \# 7 \overline{\mathbb{C P}}^{2}$.}

Here, we will use the fibration for $E(1)$ described in construction 2. We will also use a double node neighborhood $D$ containing two of the fishtails of our fibration which have the same monodromy and we will perform knot surgery along a regular fiber in this neighborhood with a knot $K$ having the properties listed in [2]. The result of knot surgery will be to remove a smaller disk from the disk $E_{5}$, which is a section in our original picture, and to replace it with the Seifert surface of $K$. This will give us a pseudo-section, that is a disk with a positive double point in $H_{2}\left(D_{k}, \partial ; \mathbb{Z}\right)$ and, after 4 blow-ups as indicated in Figure 5, an embedding of $P_{2}$ in $\mathbb{C P}^{2} \# 13 \overline{\mathbb{C P}}^{2}$ (see Figure 6). Our claim is that after blowing down we will get a manifold $X_{3}^{\prime}$ homeomorphic to $\mathbb{C P}^{2} \# 7 \overline{\mathbb{C P}}^{2}$. Using disks on a fishtail fiber and on the exceptional spheres $E_{10}$ and $E_{12}$, one can prove that $\pi_{1}\left(\mathbb{C P}^{2} \# 13 \overline{\mathbb{C P}}^{2}-P_{2}\right)$ is trivial. The rest of the argument is very similar to the proofs of Theorems 7 and 11 above and is therefore left as an exercise for the reader.

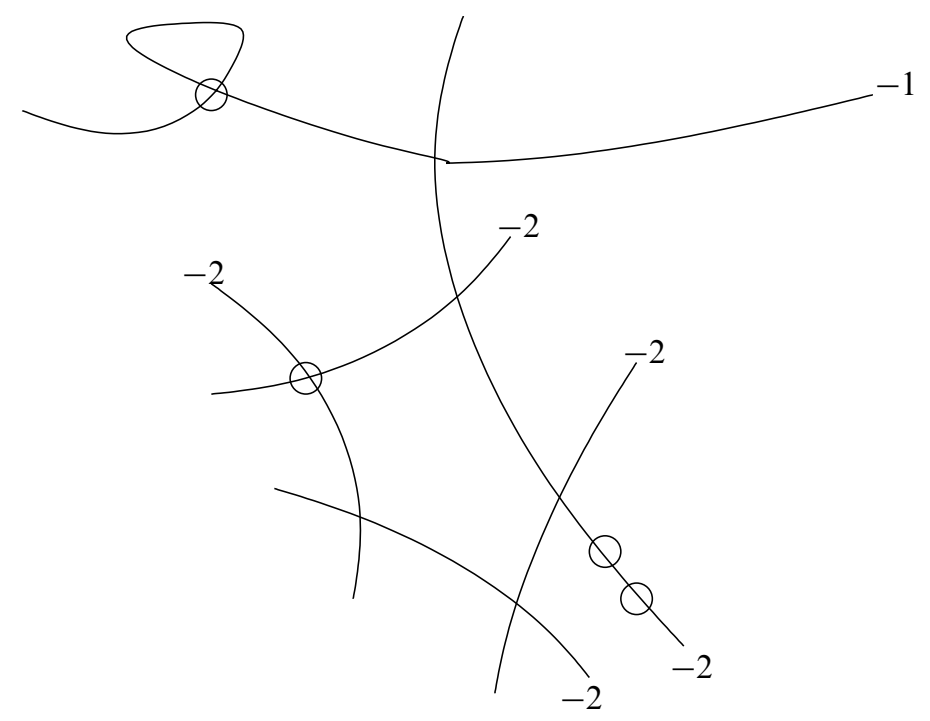

Figure 5: The 4 blow-ups performed in construction 3

Another example $\left(X_{3}^{\prime \prime}\right)$ The reader may have already noticed that it could be possible to construct an exotic smooth structure on $\mathbb{C P}^{2} \# 7 \overline{\mathbb{C P}}^{2}$ by blowing down the next plumbing tree in our family, that is $M(0 ;(1,1),(3,2),(7,6),(11,2))(p=q=2$, $r=6)$ in Neumann's notation, inside $\mathbb{C P}^{2} \# 15 \overline{\mathbb{C P}}^{2}$. 
This alternative construction can indeed be carried out using a fibration of $E(1)$ with an $I_{7}$ fiber and five fishtail fibers. We give a short outline of this construction by indicating that the double points of three of the fishtail fibers will be blown up so that after smoothing out the intersection points of these fibers with the section we can get a -7 sphere. One of the two remaining fishtail fibers will then be used in proving simple connectivity of the complement of the plumbing tree in $\mathbb{C P}^{2} \# 15 \overline{\mathbb{C P}}^{2}$.

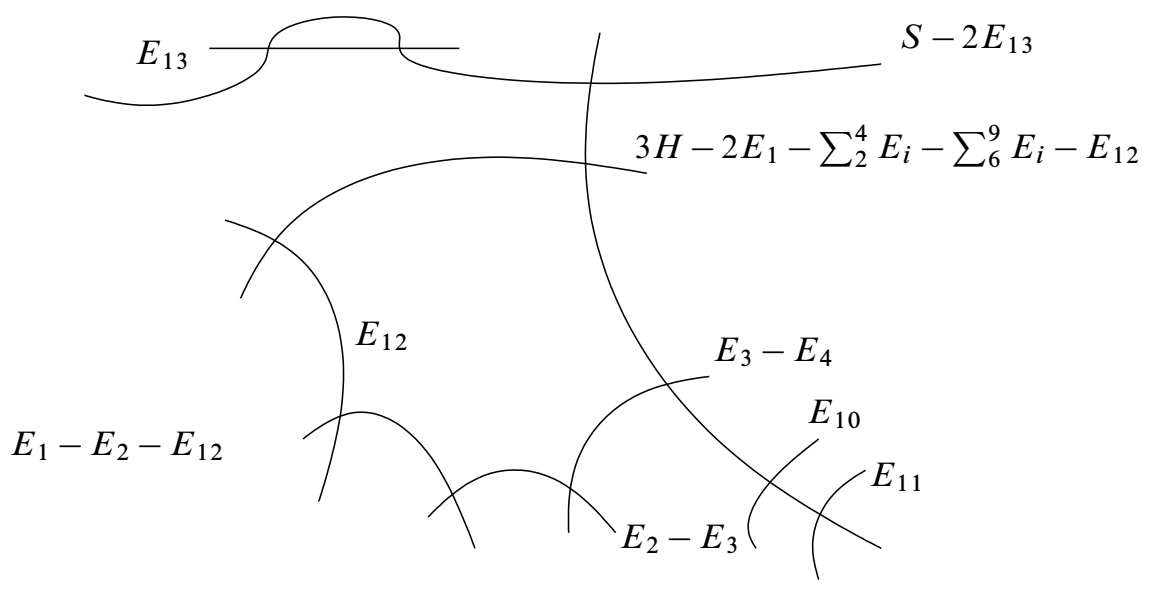

Figure 6: $P_{2}$ in $\mathbb{C P}^{2} \# 13 \overline{\mathbb{C P}}^{2}$

Remark 12 A question most naturally arising here is whether $X_{3}^{\prime \prime}$ is a member of the family of $X_{3}^{\prime}$ 's or not.

\subsection{An exotic smooth structure on $\mathbb{C P}^{2} \# 6 \overline{\mathbb{C P}}^{2}$.}

Let

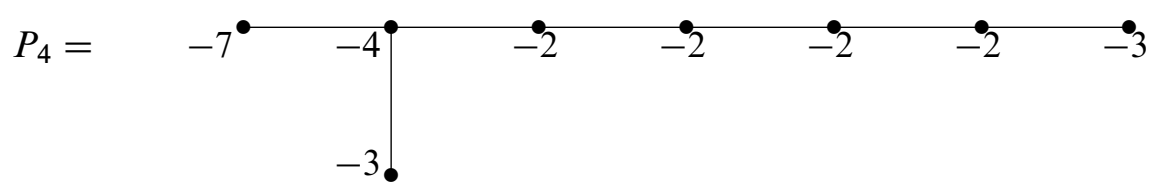

At this point, we only briefly note that starting with a fibration of $E(1)$ with one $I_{7}$ fiber and five fishtail fibers and performing knot surgery along a regular fiber in a double node neighborhood (as in 3.3) together with five appropriate blow-ups we get an embedding of $P_{4}$ in $\mathbb{C P}^{2} \# 14 \overline{\mathbb{C P}}^{2}$. Along the lines of our previous arguments, it can be proven that $P_{4}$ bounds a rational homology ball and that blowing down along $\partial P_{4}$ gives a manifold $X_{4}^{\prime}$ homeomorphic to $\mathbb{C P}^{2} \# 6 \overline{\mathbb{C P}}^{2}$.

Algebraic $8 \mathcal{G}$ Geometric Topology, Volume 7 (2007) 


\section{Computation of Seiberg-Witten invariants}

Theorem 13 There is a characteristic cohomology class $K_{i}^{\prime} \in H^{2}\left(X_{i}^{\prime}\right)$ such that $S W_{X_{i}^{\prime}}\left(K_{i}^{\prime}\right) \neq 0$.

Corollary 14 The 4-manifold $X_{i}^{\prime}$ is not diffeomorphic to $\mathbb{C P}^{2} \#(10-i) \overline{\mathbb{C P}}^{2}$.

Proof It is known that the SW invariants of $\mathbb{C P}^{2} \#(10-i) \overline{\mathbb{C P}}^{2}, i \in\{1,2,3,4\}$, are trivial, because of the existence of a metric with positive scalar curvature. This, combined with Theorem 13 and the fact that the SW invariants are diffeomorphism invariants, leads to a proof of the corollary.

Proof of Theorem 13 We will apply Theorem 3 to all four constructions.

(i) First construction:

Denote $\mathbb{C P}^{2} \# 13 \overline{\mathbb{C P}}^{2}$ by $X_{1} . \quad Y_{1}$ is a monopole L-space (see Kronheimer, Mrowka, Ozsváth and Szabó [9] for a proof) and $\left|H_{1}\left(Y_{1}\right)\right|=81$. In addition, $B_{1}$ and $P_{1}$ are negative definite 4-manifolds. Consider

$$
K_{1} \in H^{2}\left(X_{1} ; \mathbb{Z}\right), K_{1}(H)=3, K_{1}\left(E_{i}\right)=1, i \in 1,2, \ldots, 13
$$

and denote $\left.K_{1}\right|_{X_{1}-\operatorname{int}\left(P_{1}\right)}$ by $K_{1 \mid} . K_{1 \mid}$ extends as a characteristic cohomology class to $X_{1}^{\prime}$ (proved using the embedding of $P_{1}$ in $\# 4 \overline{\mathbb{C P}}^{2}$ and more specifically that $K_{1}$ evaluates on the spheres of $P_{1}$ in the same way that the canonical class of $\# 4 \overline{\mathbb{C P}}^{2}$ evaluates on them). Denote this extension of $K_{1 \mid}$ by $K_{1}^{\prime}$. Finally, consider

$$
a_{1}=6 H-2 E_{1}-2 E_{2}-\sum_{i=4}^{9} 2 E_{i}-E_{10}-E_{12}-E_{13} .
$$

Note that for such an $a_{1}$, the following conditions hold:

$a_{1} \in H_{2}\left(\mathbb{C P}^{2} \# 13 \overline{\mathbb{C P}}^{2} ; \mathbb{Z}\right), a_{1} \cdot a_{1} \geq 0, H \cdot a_{1}>0, K_{1}\left(a_{1}\right)<0, a_{1}$ is represented in $\left(\mathbb{C P}^{2} \# 13 \overline{\mathbb{C P}}^{2}-\operatorname{int}\left(P_{1}\right)\right)$.

Now $H \cdot a_{1}>0$ and $K_{1}\left(a_{1}\right)<0$ imply the existence of a wall between $\operatorname{PD}(H)$ and $\operatorname{PD}\left(a_{1}\right)$. In the chamber corresponding to $\operatorname{PD}(H), S W_{X_{1}}$ is trivial, since we have a positive scalar curvature metric. The wall crossing formula implies therefore that $S W_{X_{1}, a_{1}}\left(K_{1}\right)= \pm 1$.

By the dimension formula (2), $d\left(K_{1}\right)=0$ and $d\left(K_{1}^{\prime}\right)=0$ since $d$ remains unchanged by our operation. It follows from the preceding analysis that we can apply Theorem 3 to our case and thus get that

$$
S W_{X_{1}^{\prime}, a_{1}}\left(K_{1}^{\prime}\right)= \pm 1
$$


which completes the proof of Theorem 11 for our first construction.

Note that there is no ambiguity about the chambers in the blown down manifold, since the wall crossing formula combined with the dimension formula for SW invariants implies that for a 4-manifold $\mathrm{M}$ with $b_{2}^{+}(M)=1$ and $b_{2}^{-}(M) \leq 9$ there is only one chamber.

(ii) Second construction:

Asking for the analogous to the above conditions to be fullfilled, one can easily compute that

$$
a_{2}=7 h-3 e_{1}-2 \sum_{2}^{9} e_{i}-e_{12}-e_{13}-2 e_{14}
$$

is a cohomology class that can be used for the computation of the Seiberg-Witten invariants in this case.

(iii)-(iv) In a similar fashion, one can carry out the computations for the remaining cases.

Note 15 D Gay and A Stipsicz recently proved in [4] that Wahl type diagrams provide examples of plumbing trees that can be symplectically blown down. Making use of their results, it follows immediately that the manifolds $X_{1}^{\prime}, X_{2}^{\prime}$ and $X_{3}^{\prime \prime}$ constructed above are symplectic.

\section{Appendix A Elliptic fibrations of $E(1)$}

We give explicit constructions for some of the elliptic fibrations $E(1) \rightarrow \mathbb{C P}^{1}$ used in the paper. Note that the existence of such fibrations can also be verified using the monodromies of the singular fibers.

\section{A.1 A fibration of $E(1)$ with a singular fiber of type $I_{3}$ and nine fishtail fibers}

Let $C_{1}$ and $C_{2}$ be two complex curves in the complex projective plane, such that $C_{2}=\left\{[x: y: z] \in \mathbb{C P}^{2} \mid p_{2}(x, y, z)=x^{3}+z x^{2}-z y^{2}=0\right\}$ or any curve isotopic to this so that it will give rise to a fishtail fiber in $E(1), C_{1}$ is the union of three lines $L_{1}, L_{2}, L_{3}$ - defined by an equation of the form $p_{1}(x, y, z)=0$ and $C_{1}, C_{2}$ intersect as indicated in Figure 7.

Algebraic $8 \mathcal{G}$ Geometric Topology, Volume 7 (2007) 


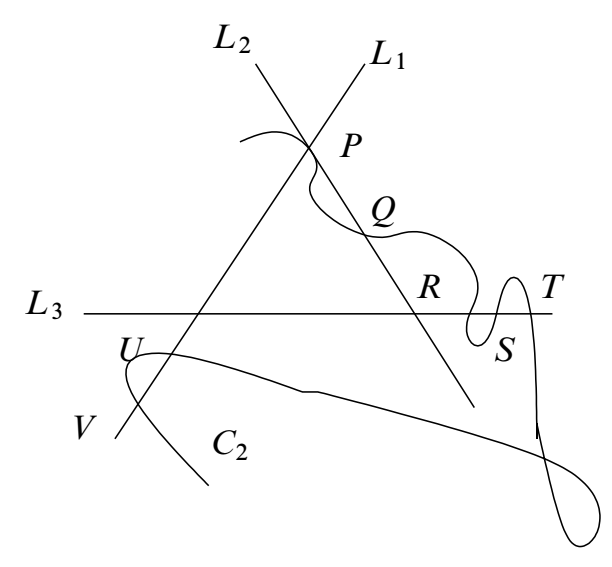

Figure 7: Curves generating the pencil

Perform three infinitely close blow-ups at the base point $P$ and six further blow-ups at the base points $Q, R, S, T, U, V$. After doing so, the curves $C_{1}$ and $C_{2}$ get locally separated and the pencil of elliptic curves

$$
C_{t}=C_{\left[t_{1}: t_{2}\right]}=\left\{\left(t_{1} p_{1}+t_{2} p_{2}\right)^{-1}(0)\right\},\left[t_{1}: t_{2}\right] \in \mathbb{C P}^{1}
$$

provides a well defined map $\mathbb{C P}^{2} \# 9 \overline{\mathbb{C P}}^{2} \rightarrow \mathbb{C P}^{1}$. It is easy to check that starting with the curves $C_{1}, C_{2}$ and performing the nine blow-ups as described above, one can detect an $I_{3}$ singular fiber, a fishtail fiber and $E_{3}$ as a section (See also Figure 8). Using the equations defining $C_{1}$ and $C_{2}$, the remaining singular fibers can be determined as well.

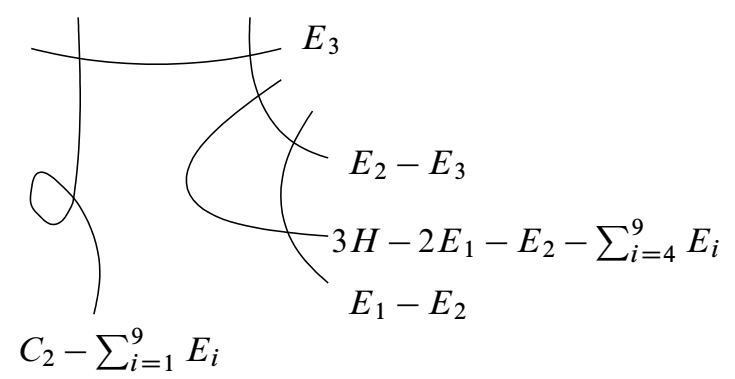

Figure 8: A section, a fishtail and an $I_{3}$ fiber in $E(1)$

\section{A.2 A fibration of $E(1)$ with a singular $I_{5}\left(I_{7}\right)$ fiber and seven (five) fishtail fibers.}

Such fibrations can also be easily constructed starting with appropriate generating curves for the pencil.

Algebraic $8 \mathcal{G}$ Geometric Topology, Volume 7 (2007) 
Even more easily, one can verify the existence of such fibrations combinatorially, using their monodromy. We will do so for one of the two cases.

According to Kodaira [5; 6; 7], the monodromies of our singular fibers are as follows:

The monodromy of the fishtail fiber $I_{1}$ is $a=\left(\begin{array}{ll}1 & 1 \\ 0 & 1\end{array}\right)$ and the monodromy of the singular fiber $I_{k}$ is $\left(\begin{array}{ll}1 & k \\ 0 & 1\end{array}\right)$. If $b=\left(\begin{array}{cc}1 & 0 \\ -1 & 1\end{array}\right)$, then $b=(a b) a(a b)^{-1}$, and so $b$ also represents the monodromy of a fishtail fiber.

In addition, $\left(a^{3} b\right)^{3}=I \Leftrightarrow a^{5}\left(a^{-2} b a^{2}\right) a b a a a b=I$, which means that the fibration with an $I_{5}$ and seven fishtail fibers over the disk extends to a fibration over $S^{2}$. The classification of genus-1 Lefschetz fibrations shows that the resulting fibration is an elliptic fibration on $E(1)$.

\section{References}

[1] R Fintushel, R J Stern, Rational blowdowns of smooth 4-manifolds, J. Differential Geom. 46 (1997) 181-235 MR1484044

[2] R Fintushel, R J Stern, Double node neighborhoods and families of simply connected 4-manifolds with $b^{+}=1$, J. Amer. Math. Soc. 19 (2006) 171-180 MR2169045

[3] M H Freedman, The topology of four-dimensional manifolds, J. Differential Geom. 17 (1982) 357-453 MR679066

[4] D Gay, A Stipsicz, Symplectic rational blow-down along Seifert fibred 3-manifolds arXiv: math.SG/0703370

[5] K Kodaira, On compact analytic surfaces, from: "Analytic functions", Princeton Univ. Press, Princeton, N.J. (1960) 121-135 MR0140519

[6] K Kodaira, On compact analytic surfaces II, Ann. of Math. (2) 77 (1963) 563-626 MR0184257

[7] K Kodaira, On compact analytic surfaces III, Ann. of Math. (2) 78 (1963) 1-40 MR0184257

[8] P Kronheimer, T Mrowka, Floer homology for Seiberg-Witten monopoles, in preparation (2005)

[9] P Kronheimer, T Mrowka, P Ozsváth, Z Szabó, Monopoles and lens space surgeries, Ann. of Math. (2) 165 (2007) 457-546 MR2299739

[10] J W Morgan, The Seiberg-Witten equations and applications to the topology of smooth four-manifolds, Mathematical Notes 44, Princeton University Press, Princeton, NJ (1996) MR1367507

[11] W D Neumann, An invariant of plumbed homology spheres, from: "Topology Symposium, Siegen 1979 (Proc. Sympos., Univ. Siegen, Siegen, 1979)”, Lecture Notes in Math. 788, Springer, Berlin (1980) 125-144 MR585657 
[12] P Ozsváth, Z Szabó, On Park's exotic smooth four-manifolds, from: “Geometry and topology of manifolds”, Fields Inst. Commun. 47, Amer. Math. Soc., Providence, RI (2005) 253-260 MR2189937

[13] J Park, Simply connected symplectic 4-manifolds with $b_{2}^{+}=1$ and $c_{1}^{2}=2$, Invent. Math. 159 (2005) 657-667 MR2125736

[14] J Park, A I Stipsicz, Z Szabó, Exotic smooth structures on $\mathbb{C P}^{2} \# 5 \overline{\mathbb{C P}}^{2}$, Math. Res. Lett. 12 (2005) 701-712 MR2189231

[15] A Scorpan, The wild world of 4-manifolds, Amer. Math. Soc., Providence, RI (2005) MR2136212

[16] A Stipsicz, $\mathbf{Z}$ Szabó, An exotic smooth structure on $\mathbb{C P}^{2} \# 6 \overline{\mathbb{C P}}^{2}$ arXiv: math.GT/0411258

[17] J Wahl, Smoothings of normal surface singularities, Topology 20 (1981) 219-246 MR608599

Department of Mathematics, Princeton University

Fine Hall, Washington Road, Princeton NJ 08544, USA

mariam@math.princeton.edu

Received: 11 October $2006 \quad$ Revised: 16 April 2007 